\title{
Spectral Analysis of the Efficiency of Vertical Mixing in the Deep Ocean due to Interaction of Tidal Currents with a Ridge Running down a Continental Slope
}

\author{
R. N. Ibragimov ${ }^{1,3 *}$, A. Tartakovsky ${ }^{1,2}$ \\ ${ }^{1}$ Pacific Northwest National Laboratory, Richland, WA 99352, USA \\ ${ }^{2}$ School of Geosciences, Department of Mathematics and Statistics \\ University of South Florida, Tampa, FL \\ ${ }^{3}$ Lead Mathematician, Applied Statistics Lab, GE Global Research 1 \\ Research Circle Niskayuna, NY 12309
}

\begin{abstract}
Efficiency of mixing, resulting from the reflection of an internal wave field imposed on the oscillatory background flow with a three-dimensional bottom topography, is investigated using a linear approximation. The radiating wave field is associated with the spectrum of the linear model, which consists of those mode numbers $n$ and slope values $\alpha$, for which the solution represents the internal waves of frequencies $\omega=n \omega_{0}$ radiating upwrad of the topography, where $\omega_{0}$ is the fundamental frequency at which internal waves are generated at the topography. The effects of the bottom topography and the earth's rotation on the spectrum is analyzed analytically and numerically in the vicinity of the critical slope

$$
\alpha_{n, \theta}^{c}=\arcsin \left(\frac{n^{2} \omega_{0}^{2}-f^{2}}{N^{2}-f^{2}}\right)^{\frac{1}{2}}
$$

which is a slope with the same angle to the horizontal as the internal wave characteristic. In this notation, $\theta$ is latitude, $f$ is the Coriolis parameter and $N$ is the buoyancy frequency, which is assumed to be a constant, which corresponds to the uniform stratification.
\end{abstract}

Keywords and phrases: internal waves, ocean mixing, effects of rotation, flows over topography

Mathematics Subject Classification: 76B55, 76B70, 37N10

\section{Introduction}

Waves produced at the sea surface and sea floor propagate into the ocean interior, generating small-scale mixing processes that affect circulation, heat transport, and nutrient distribution and, in turn, biological

${ }^{*}$ Corresponding author. E-mail: ibrranis@gmail.com 
productivity ([2]; [35]). Current estimates suggest that 40-50\% of the energy required for a deepocean mixing is injected by tide-topography interactions with the remainder coming from wind forcing. Observations by [36] based on measurements of microstructure show that mixing is considerably increased over bottom irregularities. Particularly, tidal flows result in mixing directly above the boundaries, in which case an issue of particular importance is the rate at which oceanic fluid from the interior is exchanged with fluid at the sloping boundary. According to [2], this exchange plays a paramount role in the efficiency of boundary mixing, allowing it to contribute significantly to the global overturning circulation ([11]; [17]; [18]). Stratified flows over topography are of interest for meteorology as well ([38]; [33]; [16]; [1]).

The total length of the world's continental slopes sums up to $300,000 \mathrm{~km}$. More than one-half of all continental slopes descend in deep sea trenches. The rest of the slopes descend into the shallow marine sediments (see Figure 1). It has been observed that the "Pacific Continental Slope" is steeper than the "Atlantic Continental Slope" whereas the continental slope gradients are flattest in the Indian Ocean ([26]). The continental slopes occupy almost $8.8 \%$ of the world's surface.

Our main focus of is an analyses of the vertical energy flux radiating upward into the interior of the ocean that results from the internal wave reflection from the continental slope with the superimposed prominent corrugation. The corrugation is assumed to run directly up and down the slope. We focus on the case when the topography produces only a small perturbation to the flow field. The presence of the topography generates a localized disturbance, which propagates into the ocean interior from the source region in the form of an internal gravity wave field. The ocean is idealized as vertically unbounded so that radiated energy freely escapes to $z=+\infty$. In other words, since the source of wave energy is at the bottom, all waves have upward group velocity. In the ocean, time dependent tidal currents passing over topographic bottom features are a significant source of internal waves ([28]; [45]; [12]; [24]; [9]). In this work we investigate internal wave generation due to time dependent harmonic basic flow of the form $\vec{u}_{0}(t)=\left(U_{0} \cos \left(\omega_{0} t\right), V_{0} \sin \left(\omega_{0} t\right), W_{0} \sin \left(\omega_{0} t\right)\right)$. Since Coriolis forces are dynamically significant in oceanographic applications, our model is considered in a rotating reference frame. Although the presence of an oceanic free surface is also significant ([21]]), perhaps more so than rotation, we note that if we assume that waves generated by the topography are dissipated before returning to the bottom after reflection from the free surface, our solution will be of physical reference to the real ocean. Otherwise, by assuming an infinite slope, this work does not permit exchange between mixed boundary-layer fluid and stratified ocean waters which almost certainly occurs in the real ocean (see e.g., [11], where reduced mixing efficiency arising from boundary-layer physics is actually claimed ([20]; [44]; [41]).

A simple linear solution for flow at constant velocity and constant stratification over a Witch of Agnesishaped mountain of height $H$ and half-width $L: h(x)=H L^{2} /\left(L^{2}+x^{2}\right)$ (in our model, we consider the same form of corrugation) has been investigated in [37]. More recently, [45] have given a particularly succinct review of many aspects of flow over mountains. The current knowledge of flows over hills is summarized in [13]; [3]; [19]. The tides have been reexamined in [32] as a possible source of energy for diapycnal mixing in the ocean interior. Evidence from satellite altimetry indicates that as much as $30 \%$ of tidal dissipation occurs in the open ocean ( [8]), a process previously thought to occur almost exclusively on the continental shelf. Much recent activity has therefore been focused on understanding where and how this open ocean component of tidal dissipation occurs ([6]; [3], [4]; [21]; [27]; [36]; [26]). A particular question of interest for climate studies is how much of the tidal energy is converted into potential energy through diapycnal mixing. In deep water, energy is transferred from the large scale barotropic tide to small turbulent mixing scales through a series of stages. First baroclinic motions are generated by the barotropic tidal flow over topography. Baroclinic energy is then transferred to smaller scales, leading to wavebreaking and mixing, through nonlinear wave-wave interactions or further interactions with topography. In this study we examine only one of these stages - the mixing which results when baroclinic internal tides reflect from sloping topography.

Previous studies of internal wave generation by flow over topography have identified two important controlling parameters (see e.g., [26]; [25]). The first parameter $\epsilon=|\nabla H| / s$ is a measure of the relative 


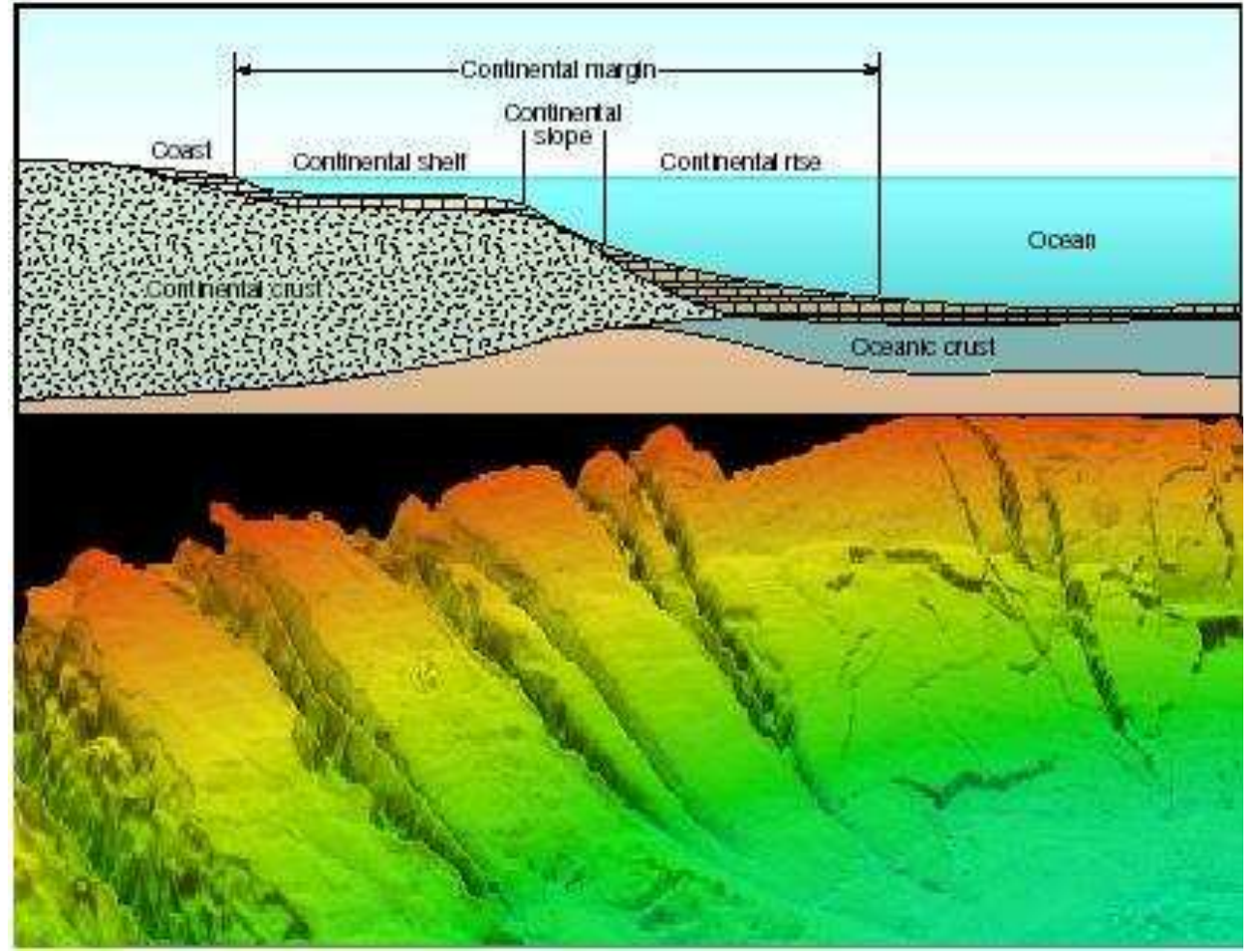

FiguRe 1. Upper panel: Schemtic presentation of a continental slope representing the submerged border gradually decreasing to the ocean bottom. Lower panel: Bird's-eye view of continental slope and rise near Hudson Canyon. Note the landslide head-scarps and lobate deposits along the rise. Image source: NGDC.

steepness of the topography, where the bottom is at $z=-H+h(x, y)$ with $H$ being the depth, and

$$
s=\frac{k}{m}=\left(\frac{\omega^{2}-f^{2}}{N^{2}-\omega^{2}}\right)^{\frac{1}{2}}
$$

is the slope of an internal wave group velocity characteristic. Here we use customary notation (see e.g., [22]) in which $k$ is the horizontal wavenumber, $m$ is the vertical wavenumber, $\omega$ is internal wave frequency, $f$ is the Coriolis frequency, and $N$ is the buoyancy frequency. As has been poined in [25], it is most likely that the most efficient mixing is resulting from the reflection of an internal wave from a slope at values $\alpha$ close to its critical angles $\alpha^{c}$ for which $\tan \alpha^{c}$ given by (1.1). The second parameter is $R_{L}=U_{0} /\left(L \omega_{0}\right)$, where $L$ is topographic length scale. This parameter is a ratio between the tidal excursion distance $U_{0} / \omega_{0}$ and the length of the topographic feature. It is one measure of nonlinearity. A third parameter is $a / H$ where $a$ is the topographic amplitude and $H$ is the water depth away from topography.

Bell, [6] considered the process of internal gravity wave generation by simple harmonic flow $u_{0}=$ $U_{0} \cos \left(\omega_{0} t\right)$ of a stratified flow over an obstacle. His analysis was for an infinitely deep ocean with thee-dimensional topography $h(x, y)$ in the limit $\epsilon<<1$, with finite $R_{L}$ (see e.g. [26], [25]). Bell's model linearizes the problem by applying the boundary condition at $z=0$, as we do here in a rotated three-dimensional coordinate system, rather than at the bottom topography $z=-H+h(x, y)$ (we show later that, in an appropriately rotated coordinate system, we can linearize the problem about $z=0$ as in Bell paper although, originally, the bottom is at $z=\alpha y$ ). Balmforth et al, [5], have extended Bell's 
theory to steeper topography $(0<\varepsilon<1)$ but their linearization is justified provided that $R_{L}<<1$, i.e., the tidal excursion is much less that the scale of the topography. Khatiwala [21] in part based on Bell, [6] examines the problem of internal wave generation by the interaction of an oscillating tidal flow with two-dimensional bottom topography $z=-H+h(x)$. Unlike Bell's theory, which is applicable for a fluid of infinite depth in which energy input at the bottom radiates upward, Khatiwala imposes an upper rigid lid boundary condition resulting in a horizontal energy flux. Thorpe, [39], [40] extended the wave generation problem to uniform flow along a corrugated slope. His solution includes the effects of the earth's rotation and he considered corrugations that are at an angle to the direction of steepest slope. MacReady 89 Pawlak, [29] presented an alternative derivation of Thorpe's solution (neglecting the effect of rotation) and extended it to flows above and below the low speed cutoff. Legg, [26] performed numerical experiments for internal tide generation for a continental slope characterized by ridges and valleys running up and down the slope. Other related studies by Kunze, [23] and Nash E3 Moum, [34] have considered internal tides on the continental slope and internal hydraulic flows on the continental shelf, and their results are not directly applicable to the deep ocean processes considered here. The process of generation of internal waves due to tide/topography interactions associated with oscillating along-isobath currents impinging on a ridge running down a slope with an inclusion of large tidal excursion to generate harmonics has been considered in Ibragimov, [14]. The experimental work confirming some of the analytic predictions from [14] have been reported recently in [15].

This work is an extension of the work in Ref. [14]. Here we focus on the analysis of the harmonics and the effects of rotation on the energy flux in the vicinity of the critical slope $\alpha_{c}$.Additionally, we also analyze the effects of concavity of the corrugation on the resulting energy flux which has also not been analyzed in [14]. Apparently, increasing the horizontal scale $L$ of the corrugation while the vertical scale $H$ is being fixed, leads to the change of the concavity of the corrugation. Thus this work combines that of [6] for harmonics generated by large tidal excursion over weak topography (small-amplitude $h / H<<1$, gentle subcritical slope $h_{x} / s<<1$ ) on a flat bottom with recent extensions on a slope ([39]; [29]). Unlike these latter authors, here we primarily focus on the effects of rotation and the scales of the corrugation of internal wave generation due to harmonic flow with the goal of estimating the flux of energy into the internal wave field in the vicinity of the critical slope.

\section{Spectrum of the model}

For the sake of definiteness, we set the axes as: $x^{\prime}$ (assumed eastward), $y^{\prime}$ (northward) and $\widehat{k}^{\prime}$ is the unit vector in the vertical $z^{\prime}$ direction (opposite gravity). The fluid velocity is $\vec{u}=(u, v, w)$ relative to the Cartesian coordinate system $\left(x^{\prime}, y^{\prime}, z^{\prime}\right)$. Motion is considered to be outside of the frictional boundary layers, allowing viscosity to be neglected. In the theory of ocean circulation and within meteorological applications, it is commonplace to make the Boussinesq approximation in which the full variation of density is retained only in the buoyancy force in the vertical momentum balance. Wherever else it occurs, in the horizontal momentum balance, and in the continuity equation, density is replaced by a constant (in the simplest implementation). In particular, this means that the three-dimensional velocity field is assumed to be solenoidal. The analysis is applied to geophysical flows where the effect of rotation due to Coriolis force is a prominent feature. Thus within the Boussinesq approximation, the governing equations of motion for internal waves, observed in a system of coordinates rotating with angular velocity $\vec{\Omega}$ are written in the form (see e.g., [13]):

$$
\begin{aligned}
\rho_{0}\left[\frac{\partial \vec{u}}{\partial t}+\vec{u} \cdot \nabla \vec{u}+2 \vec{\Omega} \times \vec{u}\right] & =-\nabla p-g \rho \widehat{k}^{\prime}, \\
\frac{\partial \rho}{\partial t}+\vec{u} \cdot \nabla \rho+w \frac{d \bar{\rho}}{d z^{\prime}} & =0, \\
\nabla \cdot \vec{u} & =0,
\end{aligned}
$$


where $g$ is the acceleration due to gravity so that $p$ and $\rho$ are to be interpreted as the pressure and density departures from their mean state

$$
\bar{\rho}\left(z^{\prime}\right)=-\frac{\rho_{0}}{g} N^{2} z^{\prime}, \quad \bar{p}\left(z^{\prime}\right)=p_{0}-\rho_{0} g z^{\prime}-g \int_{0}^{z^{\prime}} \bar{\rho}(\xi) d \xi,
$$

in which $\rho_{0}$ is the constant reference density, $\bar{\rho}\left(z^{\prime}\right)$ is a background stable density profile with the associated buoyancy frequency $N$ defined by

$$
N^{2}=-\frac{g}{\rho_{0}} \frac{d \bar{\rho}}{d z^{\prime}}
$$

and we require $\rho_{0}+\bar{\rho}$ and $\bar{p}$ to be consistent with the state of rest, i.e.,

$$
\frac{d \bar{p}}{d z^{\prime}}=-\left(\rho_{0}+\bar{\rho}\right) g
$$

The quantity $N$, which is assumed to be a constant in the frame of the present study, measures the degree of density stratification of a fluid with average potential density $\bar{\rho}(z)$ and thus represents the frequency with which a vertically displaced fluid element would be expected to oscillate because of restoring buoyancy forces.

The traditional $f$-plane approximation is made whereby we take $2 \vec{\Omega}=(0,0, f)$, where $f$ is the inertial frequency which depends on the rotation rate of the earth (angular velocity $\Omega=2 \pi \mathrm{rad} /$ day $\approx 0.73 \times 10^{-4}$ $\left.\mathrm{s}^{-1}\right)$.

The oscillatory three-dimensional time-dependent background flow over a uniform flat slope $z=\gamma y$ is given by

$$
\vec{u}_{0}=\left(U_{0} \cos \left(\omega_{0} t\right), V_{0} \sin \left(\omega_{0} t\right), \gamma V_{0} \sin \left(\omega_{0} t\right)\right),
$$

where $\gamma=\tan \alpha$, the cross-shelf velocity $U_{0}$ is assumed to be constant and $\omega_{0}$ the frequency. Since we are mostly interested in tidal flows, hereafter we consider flows forced at $M_{2}$ tidal frequency, $\omega_{M_{2}}$ :

$$
\omega_{0}=\omega_{M_{2}} \sim 1.4 \times 10^{-4} \mathrm{~s}^{-1} .
$$

Thus the oscillating flow (2.7) is driven by the barotropic tide.

Since waves are generated not only at the fundamental frequency but also at all of its harmonics $\omega_{n}=n \omega_{0}$ less than $N$, our analysis will include, in general, an infinite number of discrete internal wave modes satisfying the dispersion relationship for internal waves. However, we will be interested only in the radiating part of the solution so that the mode numbers will be limited.

The absence of a sea surface means that our solution will be of physical reference to the real ocean only if waves generated by the topography are dissipated before returning to the bottom after reflection from the surface. This is consistent with an assumption of infinitely deep ocean. However, due to the vertical motion the background density $\bar{\rho}$ is a function of $z^{\prime}$ and $t$, i.e.,

$$
\bar{\rho}=\bar{\rho}\left(z^{\prime}-\zeta(t)\right)=\rho_{0}-\frac{N^{2}}{g}\left(z+V_{0} \frac{\gamma}{\omega} \cos \left(\omega_{0} t\right)\right),
$$

in which $\zeta$ is the vertical displacement associated with the background flow. The pressure field can then be deduced from the momentum equations (2.1) from which we find

$$
\begin{aligned}
\bar{p}= & \rho_{0}\left(f V_{0}+\omega_{0} U_{0}\right) x^{\prime} \sin \left(\omega_{0} t\right)-\rho_{0}\left(\omega_{0} V_{0}+f U_{0}\right) y \sin \left(\omega_{0} t\right)-\rho_{0} g z^{\prime}+ \\
& \frac{\rho_{0} \gamma V_{0}}{\omega_{0}}\left(N^{2}+\omega_{0}^{2}\right) z^{\prime} \cos \left(\omega_{0} t\right)+\frac{\rho_{0}}{2} N^{2} z^{\prime} .
\end{aligned}
$$

The cases $\bar{p}_{x^{\prime}}=0$ and $\bar{p}_{y^{\prime}}=0$ represent barotropic tides directly across and along the slope respectively (see Fig. 2 for the topographic coordinates). 
Small perturbations are introduced:

$$
u=u_{0}+\epsilon \widehat{u}, v=v_{0}^{\prime}+\epsilon \widehat{u}, \quad w=w_{0}^{\prime}+\epsilon \widehat{w}, p=\bar{p}+\epsilon \widehat{p}, \quad \widehat{\rho}=\bar{\rho}+\epsilon \widehat{\rho},
$$

where $\epsilon \ll 1$.

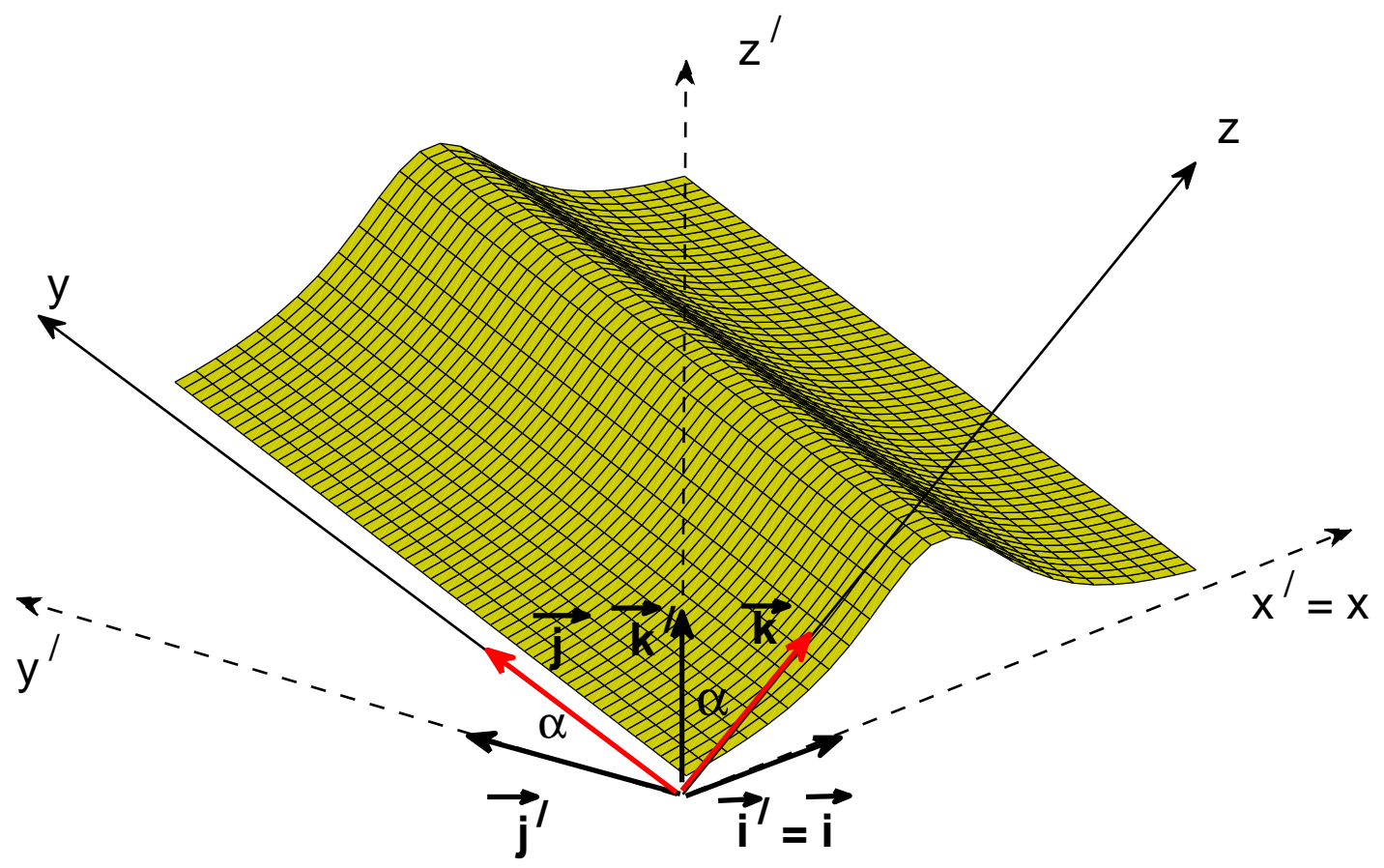

Figure 2. Corrugated slope sketch. To accommodate a sloping ocean floor, the model is considered in the system of coordinates rotatwed about the $x$ - axis by the slope angle $\alpha$, so that in the new coordinate system the $y$ - axis is directed upslope and $z$ is perpendicular to the slope.

It has been shown in the previous studies in Ref. [14] that, in the system of coordinates that is moving with the background flow $u_{0}=U_{0} \cos \left(\omega_{0} t\right)$ and that is rotated about the $x$-axis by the slope angle $\alpha$, the radiating part of the linearized model for small perturbations introduced by (2.11) is written in terms of the vertical velocity as

$$
w(x, z, t)=\sum_{|n| \in S_{N}} \int_{-\infty}^{\infty} \widehat{w}_{n}(k, z, t) d k,
$$

where,

$$
\widehat{w}_{n}=\frac{i n \omega_{0}}{2 \pi} \widehat{h}(k) J_{n}\left(\frac{k U_{0}}{\omega_{0}}\right) \exp \left[i\left(k \xi+\mu_{n} z+n \omega_{0} t\right)\right]
$$

in which

$$
\xi(x, t)=x-\int_{0}^{t} u_{0}(\tau) d \tau
$$


is the horizontal coordinate in a reference frame moving with the background flow in the rotated coordinate system, $\widehat{h}(k)$ is the Fourier transform of the topography $h(x)$ and $J$ is the Bessel function of the first kind. In the expression (2.13), the parameter $\mu_{n}$ is determined from the radiation condition as

$$
\mu_{n}=\frac{n|k|}{|n|}\left(\frac{N^{2}-n^{2} \omega_{0}^{2}}{n^{2} \omega_{0}^{2}-\widehat{f}^{2}}\right)^{\frac{1}{2}}
$$

in which

$$
\widehat{f}^{2}=N^{2} \sin ^{2} \alpha+f^{2} \cos ^{2} \alpha
$$

is a notation.

Since the generated waves are independent of the upslope coordinate $y$, the wavevectors must be in $x, z$ plane. Two such vectors exist for sufficiently small $\alpha$. Thus, as has been justified in Ref. [14], the radiating waves exist for frequencies $\omega_{n}=n \omega_{0}$, where

$$
|n| \in S_{N}=\left[n_{f}, n_{N}\right]
$$

and

$$
n_{N}=\left[\frac{N}{\omega_{0}}\right], n_{f}=\left[\frac{\widehat{f}}{\omega_{0}}\right]+1,
$$

where here $[x]$ means the greatest integer less that or equal to $x$. Particulalry, $n_{N}=7$ for $\omega_{0}=\omega_{M_{2}}$.

Particularly, if $\omega_{n}^{2}<\widehat{f}^{2}$ or $\omega_{n}^{2}>N^{2}$ the waves are evanescent. As follows from the dispersion relation of internal waves (see also Eq. (1.1)), waves of frequency $\omega_{n}$ lying between $f$ and $N$ can propagate freely only for angles

$$
\alpha<\arcsin \left(\frac{n^{2} \omega_{0}^{2}-f^{2}}{N^{2}-f^{2}}\right)^{\frac{1}{2}}
$$

which means that $\alpha$ is fixed by some harmonic of the high-frequency wave. This means that waves of frequency $\omega_{n}$ can exist only if the slope angle $\alpha=\alpha_{n \theta}$ is such that

$$
\sigma_{n, f}=\omega_{n}^{2}-\widehat{f}^{2}>0 .
$$

This is also illustrated in in Figure 3, which shows the set of critical slopes $\alpha_{n \theta}$ associated with mode numbers $n_{N} \in[1,7]$ and latitude $\theta \in\left[1^{\circ}, 89^{\circ}\right]$ North with stepsize $\Delta \theta=10$. Namely, the value $\sigma_{n, f}$ corresponding to limiting values of latitude $\theta=0^{\circ}$ an $\theta=89^{\circ}$ North are plotted by the red and blue lines respectively whereas the black dashed lines correspond to $\sigma_{n, f}$ for intermediate values of latitude. For visualization purposes, the behavior of the first mode (fundamental frequency) of $\sigma_{1, f}$ is shown separately on the upper right corner of in Figure 3. As we can see from Figure 3, as $\alpha$ increases, successively higher harmonic waves are eliminated from the wave field.

Additionally, the behavior of $\sigma_{n, f}$ versus latitude is shown in Figure 4. Although no much variation can be seen in this figure, we can still observe that the behavior of the first mode value of $\sigma_{1, f}$ differs significantly from the higher mode values of $\sigma_{n, f}, n>1$, which can also see in Figure 4 . This is also illustrated on more detailed upper frame of Figure 4 for $\sigma_{1, f}$ and $\sigma_{7, f}$.

So, the spectrum of the model consists of those mode numbers $n$, that satisfy $(2.17)$ and those angles $\alpha_{n, \theta}$, for which (2.20) holds.

\section{Identification of critical slopes and energy flux}

Within the framework of linear theory, the pressure field associated with the internal wave solution (2.12) exerts a net force

$$
F(t)=\left.\int_{-\infty}^{\infty} p(x, z, t)\right|_{z=0} \frac{d h}{d x} d x,
$$




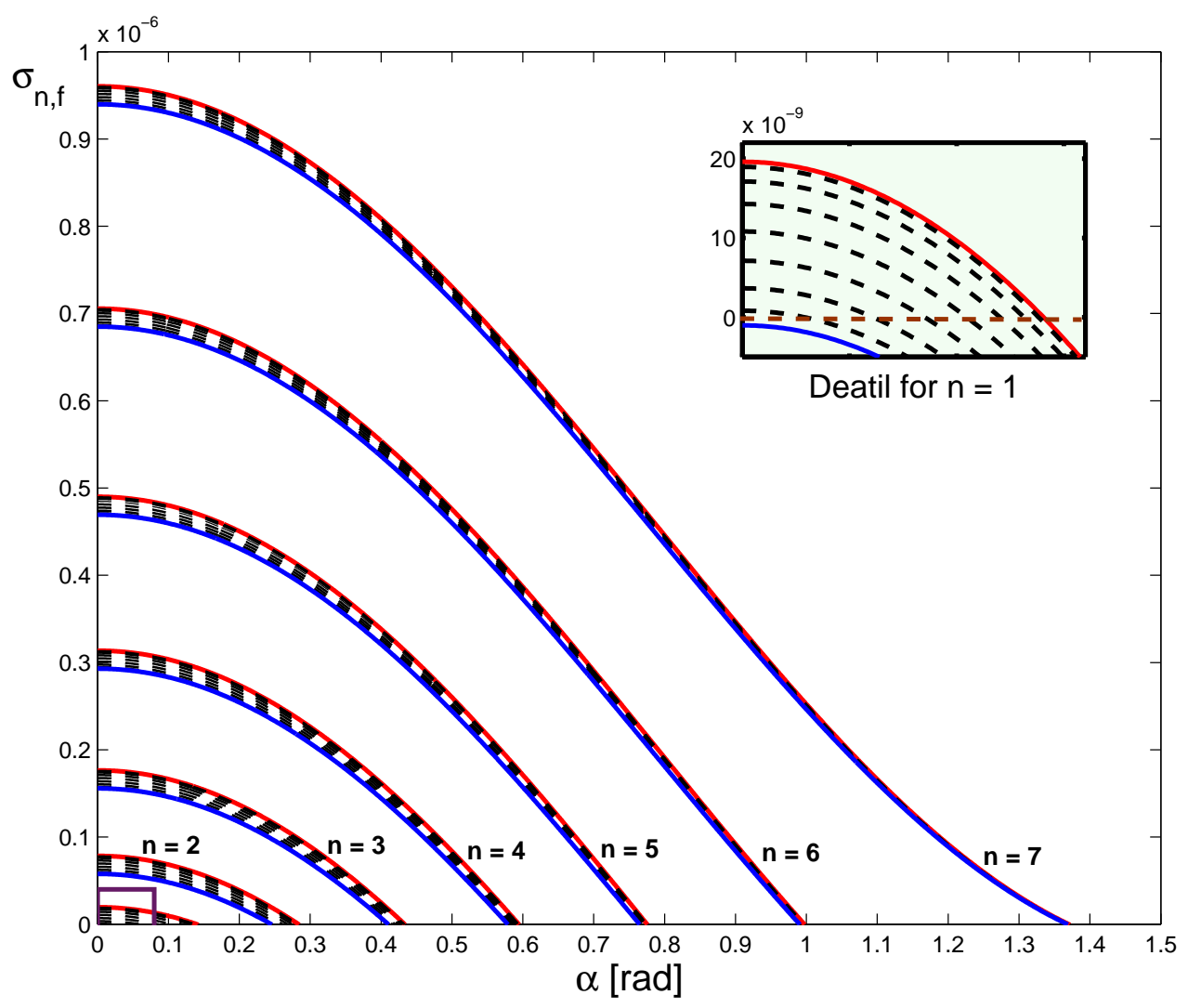

Figure 3 . Waves of frequency $\omega_{n}$ lying between $f$ and $N$ can propagate freely only for angles $\alpha$ satisfying the condition $\sigma_{n, f}=\omega_{n}^{2}-\widehat{f}^{2}>0$.

in generating the internal wave field due to the given oscillatory background flow in the vicinity of the bottom topography. Solving the linear part of the momentum equations (2.1) - (2.3), we obtain the $x$ component of the net force exerted on the topography,

$$
\begin{aligned}
F(t)= & \frac{\rho_{0}}{2 \pi} \sum_{n \in S_{N}} \int_{-\infty}^{\infty} \frac{|\widehat{h}(k)|^{2}}{k} \frac{\left(n \omega_{0}\right) \mu_{n}(k)\left(n^{2} \omega_{0}^{2}-f^{2}\right)}{n \omega_{0}+i f\left(\mu_{n} / k\right) \sin ^{2} \alpha} \\
& \times J_{n}\left(\frac{k U_{0}}{\omega_{0}}\right) \exp \left[i\left(n \omega_{0} t-\frac{k U_{0}}{\omega_{0}} \sin \omega_{0} t\right)\right] .
\end{aligned}
$$

We remark that since the power input at the bottom is radiated vertically upward, the net force $F$ can be related to the vertical energy flux $E_{z}=\langle p w\rangle$ because

$$
E_{z}=u_{0}\left\langle p \frac{d h}{d x}\right\rangle=u_{0} F
$$

As seen from the form (3.2) and as has has been illustrated in Figures 3 and $4, F(t)$ in the above expression has a singularity in the in the vicinity of the critical angles $\alpha=\alpha_{n \theta}$, i.e. the singularity 


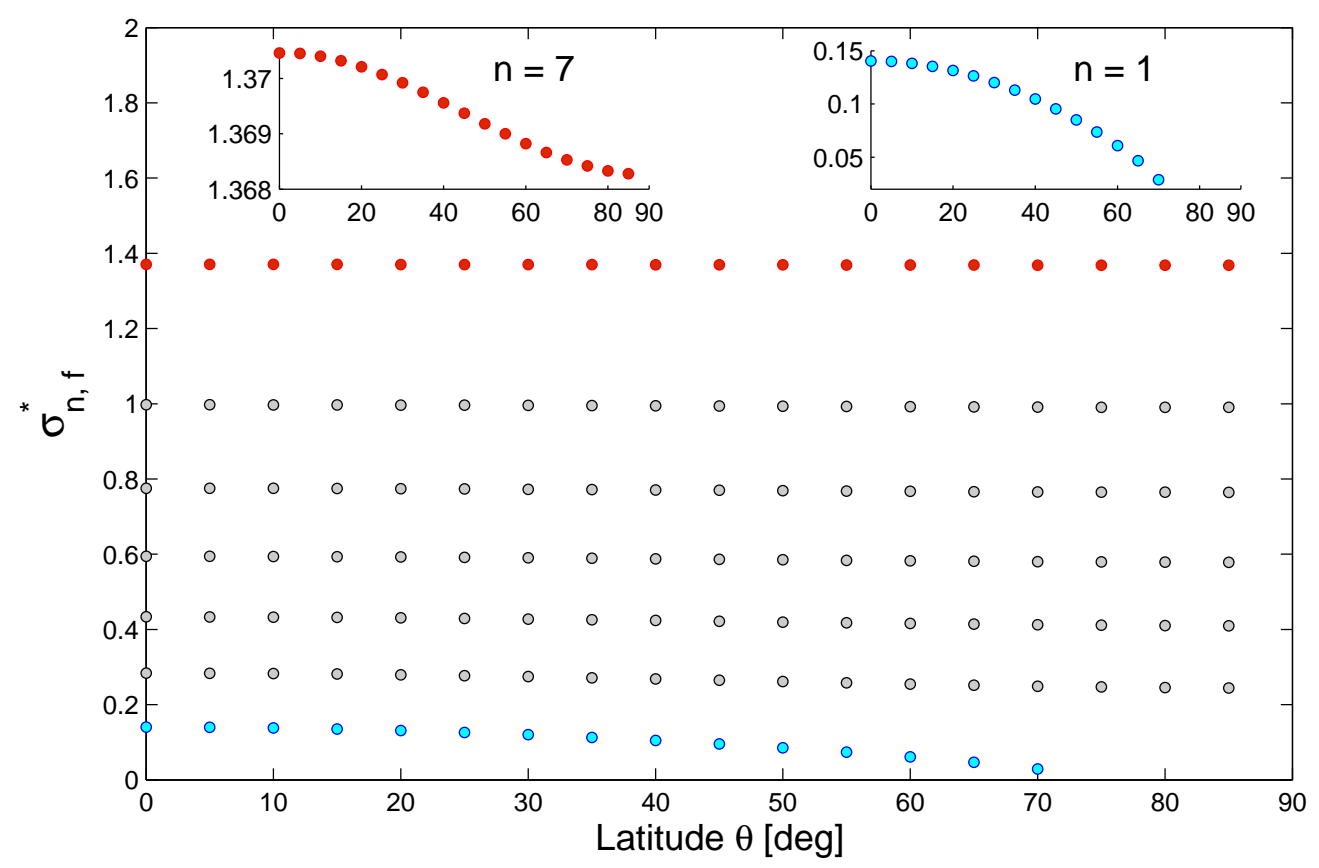

Figure 4. Destribution of zeros of $\sigma_{n, f}$ versus latitude $\theta$.

occurs if

$$
\alpha_{n \theta}=\alpha_{n \theta}^{c}=\arcsin \left(\frac{n^{2} \omega_{0}^{2}-f^{2}}{N^{2}-f^{2}}\right)^{\frac{1}{2}},
$$

which is the slope of an internal wave group velocity characteristic that has been identified in the Introduction by Eq. (1.1).

Remark: As we discussed earlier (see the discussion related to the results shown in Figures 3 and 4), radiating waves of frequency $\omega_{n}$ do exist for angles $\alpha$ satisfying the condition $\sigma_{n, f}=\omega_{n}^{2}-\widehat{f}^{2}>0$, so that we write $\alpha=\alpha_{n \theta}$ in order to indicate that the value of critical slope $\alpha^{c}$ is individual for each mode number $n$ and latitude $\theta$.

Particularly, as has been suggested by Legg, [25], a scenario that seems particularly likely to lead to mixing is the reflection of an internal wave from a slope with the same angle to the horizontal $\alpha^{c}$ as the internal wave characteristic $\tan \alpha^{c}$ given by (3.4), and $\alpha^{c}$ is known as the critical angle. It follows from our previous discussion, and also was demonstrated in Figures 3 and 4 that the singularity (and correspondingly the most efficient mixing ) occurs in the vicinity of the critical slopes defined by (3.4) and the evanescent case $\left(N^{2}<n^{2} \omega_{0}^{2}\right)$ occurs when

$$
\alpha>\alpha_{n \theta}^{c}>\arcsin \left(\frac{n^{2} \omega_{0}^{2}-f^{2}}{N^{2}-f^{2}}\right)^{\frac{1}{2}} .
$$

In this case, $\mu_{n}$ is purely imaginary. Thus evanescent modes do not contribute to $F(t)$ since, according to upward radiation of energy, $\mu_{n}$ has to be real.

To analyze the resulting net force $F$, it is advantageous to make the following three adjustments: 
(i) We average $F$ over the fundamental period $2 \pi / \omega_{0}$ and express $F$ in terms of the power input $\langle\mathcal{P}\rangle$ into the internal wave field is given by (see $[6]$ )

$$
\langle\mathcal{P}\rangle=\frac{\omega_{0}}{2 \pi} \int_{0}^{2 \pi / \omega_{0}} \operatorname{Re}\{F(t)\} u_{0}(t) d t
$$

For the comparison purposes we note that in the limiting case when $\alpha=0$ (zero slope) and $f=0$ (no rotation), the resulting time averaged power expression for $\langle\mathcal{P}\rangle$ given by (3.6) agrees with the corresponding power input given in Bell, [6]. Particularly, using (3.6), we find (see also, Ibragimov, [14]),

$$
\left.\langle\mathcal{P}\rangle\right|_{\alpha=0, f=0}=\frac{2 \rho_{0}}{\pi} \sum_{n \in S_{N}} n^{2} \omega_{0}^{2}\left(N^{2}-n^{2} \omega_{0}^{2}\right)^{\frac{1}{2}} \int_{0}^{\infty} k^{-1}|\widehat{h}(k)|^{2} J_{n}^{2}\left(\frac{k U_{0}}{\omega_{0}}\right) d k .
$$

(ii) It is convenient to normalize the power by some reference value. For definiteness, we choose the value $1 / 2 \pi \rho_{0} U_{0}^{2} N H^{2}$ which has been used in Miles, [30] wile investigating the minimization problem of the wave drag on an obstacle in the limit $H / L \rightarrow 0, U_{0} \rightarrow 0$, where $L$ and $H$ are the width and the height of the topography, respectively.

(iii) Finally, we reduce the dimension of the problem by introducing the following nondimensional parameters:

$$
\lambda=\frac{\omega_{0}}{N}, \quad \gamma=\frac{f}{N}, \quad \widetilde{k}=k L, \quad \widehat{\eta}=\frac{\widehat{h}}{L H}, \quad \beta=\frac{\sqrt{2} N L}{U_{0}}
$$

To make some comparison with the Bell's results in Ref. [6] and to analyze the effects of rotation and the topographic scales on the energy flux studied in Ibragimov, [14], we consider further a specific example when the corrugation has the form

$$
h(x)=\frac{H}{1+(x / L)^{2}},
$$

where $H$ and $L$ are respectively the characteristic height and width of the obstacle. Thus the resulting non-dimensional, normalized time average power (energy flux) in the internal wave field takes the form (hereafter we omit the symbol $^{\sim}$ )

$$
\begin{aligned}
\langle\widetilde{\mathcal{P}}\rangle= & \langle\mathcal{P}\rangle / \frac{1}{4} \pi \rho_{0} U_{0}^{2} N H^{2}= \\
& 4 \beta^{2} \sum_{n=1}^{n_{\max }} \frac{n^{3} \lambda^{3}\left(n^{2} \lambda^{2}-\gamma^{2}\right)\left(1-n^{2} \lambda^{2}\right)^{\frac{1}{2}}\left(n^{2} \lambda^{2}-\widehat{f}_{d}^{2}\right)^{\frac{1}{2}}}{\left(n^{2} \lambda^{2}-\widehat{f}_{d}^{2}\right) n^{2}+\left(1-n^{2} \lambda^{2}\right) \widehat{f}_{d}^{2} \sin ^{2} \alpha} \frac{e^{-2 \widetilde{k}}}{\widetilde{k}} J_{n}^{2}\left(\frac{\sqrt{2} \widetilde{k}}{\beta \lambda}\right) d \widetilde{k}
\end{aligned}
$$

where we denote

$$
\widehat{f}_{d}^{2}=\sin ^{2} \alpha+\gamma^{2} \cos ^{2} \alpha .
$$

As expected from the earlier discussion, the expression in (3.10) has a singularity when $\sin \alpha_{n \theta}=n \lambda$. However, this condition is met only in the evanescent case, i.e. when $\omega_{n}^{2}<N$, i.e. when

$$
\alpha_{n \theta} \geqslant \arcsin \left(\frac{n^{2} \omega_{0}^{2}-f^{2}}{N^{2}-f^{2}}\right)^{\frac{1}{2}}
$$

\section{Effects of rotation and concavity of topography on the radiating energy flux}

Further analysis is done for the fixed value of parameters $U_{0}=1.4 \times 10^{-3} \mathrm{~ms}^{-1}$ and $N=10^{-3} \mathrm{~s}^{-1}$. As seen from (3.8), for this choice of $U_{0}$, we then have

$$
\frac{U_{0}}{\sqrt{2} N} \approx 1, \quad \lambda=\frac{\omega_{M_{2}}}{N}=0.14, \quad \beta \approx L,
$$


Also, without loss of generality we can normalize the vertical scale $H$ of the topography to be equal to one. In actuality, as one can see, the parameter $H$ is not apparent in the expression (3.10) for $\langle\widetilde{\mathcal{P}}\rangle$.

To analyze the resulting power input $\langle\widetilde{\mathcal{P}}\rangle$, we first investigate the convergence of the integral

$$
Q_{n}(\beta)=\int_{\epsilon}^{M} \frac{e^{-2 \widetilde{k}}}{\widetilde{k}} J_{n}^{2}\left(\frac{\sqrt{2} \widetilde{k}}{\beta \lambda}\right) d \widetilde{k}
$$

which is the approximation of the improper integral in (3.10) for sufficiently small $\epsilon$ and sufficiently large value $M$.

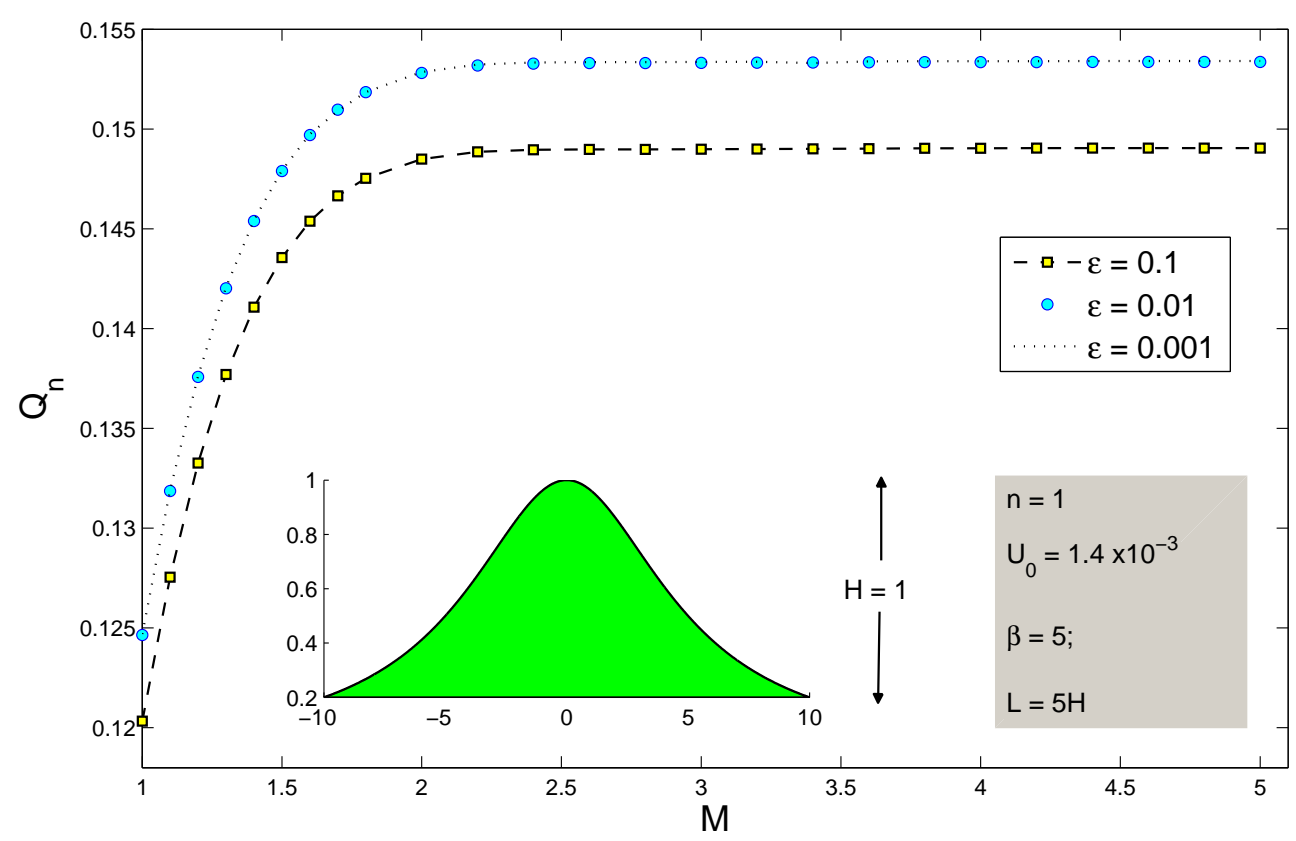

Figure 5. Convergence of the integral $Q_{n}(\beta)$ as $M \rightarrow \infty$ and $\varepsilon \rightarrow 0$.

Figure 5 is used to demonstrate the results of numerical simulations of $Q_{n}(\beta)$ versus $M$ at fixed values of $\epsilon$. We observe the numerical convergence of the integral $Q_{n}(\beta)$ as $M \rightarrow \infty$ and $\epsilon \rightarrow 0$.hereafter, in our simulations we take $M=10$ and $\varepsilon=0.001$.

Figure 6 is used to show the results of numerical simulations for the normalized power (that can also be interpreted as a vertical energy flux) $\langle\widetilde{\mathcal{P}}\rangle$ without rotational effects as a function of slope $\alpha_{n 0}$ (i.e. purely equatorial waves as observed at latitude $\theta=0^{\circ}$ North) for $L=5 H$ and the fixed values of parameters $\lambda$ and $U_{0}$ as already discussed. As we found earlier, the waves are nearly singular at the vicinity of the critical slopes $\alpha_{n \theta}^{c}$ determined by the condition (3.12), which makes the energy flux increasing rapidly at the vicinity of $\alpha_{n \theta}^{c}$. This agrees with the suggestion of Legg, [25] about an efficiency of mixing in the deep ocean due to reflection of an internal waves from critical slopes, i.e. the slopes with the same angle to the horizontal as the internal wave characteristic. Particularly, because of singular behavior $\langle\widetilde{\mathcal{P}}\rangle$ near the critical slopes $\alpha_{n 0}^{c}$, the visualization depends of stepsize of $\alpha$ and the parameter $\delta$, which characterizes 


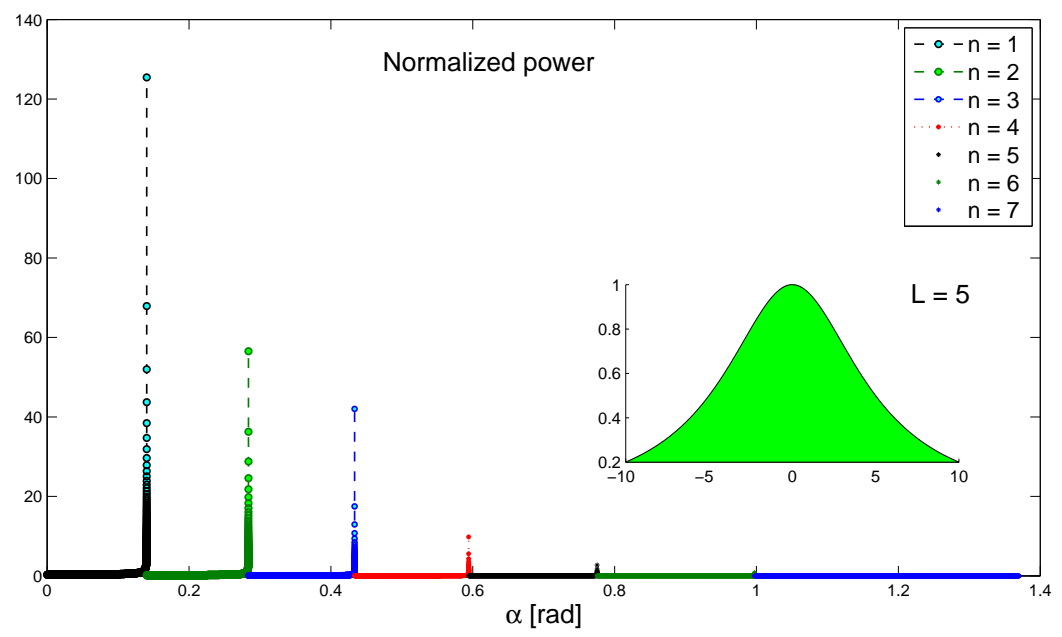

Figure 6. Vertical energy flux $\langle\widetilde{\mathcal{P}}\rangle$ as a function of slope $\alpha$ at latitude 0 deg North for $L=5$ with $\delta=0$ and stepsize $\Delta \alpha=10^{-6}$.

how close we approach to the roots $\sigma_{n, f}^{*}$ of the equation $\sigma_{n, f}=\omega_{n}^{2}-\widehat{f}^{2}=0$. We find that a quite reasonable qualitative analysis can be attained if we set, for example, $\delta=0$ and stepsize $\Delta \alpha=10^{-6}$. As we observe in Figure 6, the dominant contribution of energy distribution in internal wave field is due to waves of fundamental frequency with $n=1$. We also observe the dramatic energetic drop at slopes of $\alpha>\alpha_{n 0}^{c}$ but with subsequent "singular" increase at the succesive critical slopes $\alpha_{n 0}^{c}, n>1$.

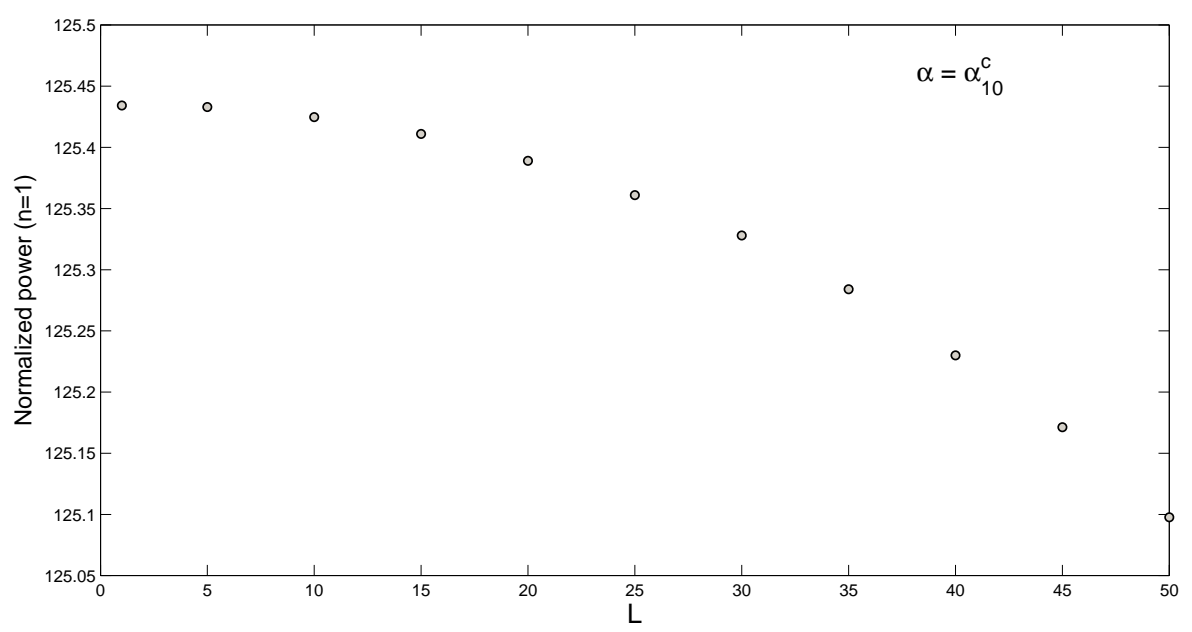

FiguRE 7. First mode normalized power at latitude $\theta=0^{\circ}$ North as a function of the characteristic horizontal scale $L$ of the corrugation in the vicinity of $\alpha=\alpha_{10}^{c}$. 

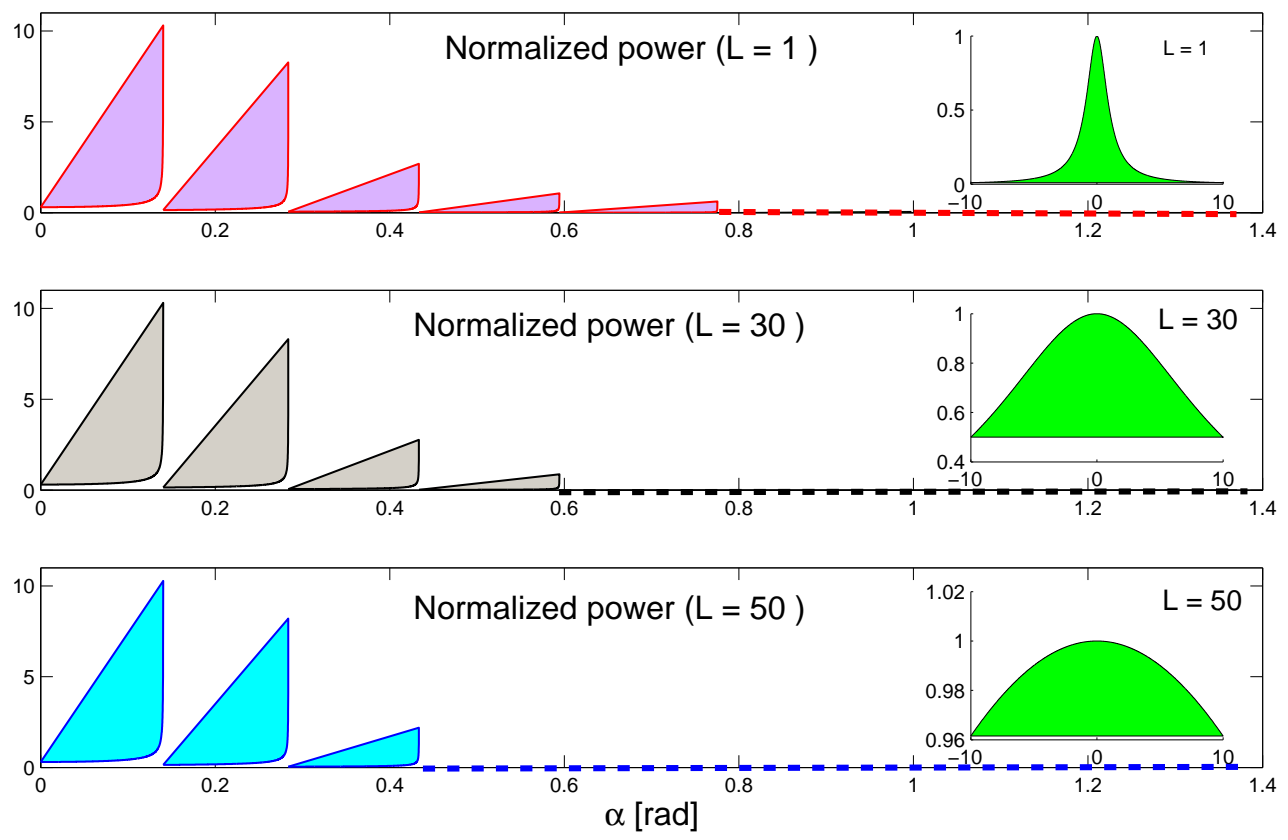

Figure 8. Qualitative energy analysis as a function of slope $\alpha$ at latitude 0 deg North with $\epsilon=0$ and stepsize $\Delta \alpha=10^{-4}$.

The effects of the topographic concavity on the normalized power $\langle\widetilde{\mathcal{P}}\rangle$ of the fundamental frequency $n=1$ is demonstrated on Figure 7 in which the normalized power is evaluated at latitude $\theta=0^{\circ}$ North (not affected by rotation). Particularly, Figure 7 is used to illustrate $\langle\widetilde{\mathcal{P}}\rangle=\left\langle\widetilde{\mathcal{P}}_{10}\right\rangle$ as a function of the horizontal topographic scale $L$ at the same values of other parameters as been used in Figure 6 . As we can see from the numerical simulations presented in this figure, as $L$ goes from 1 to 50 , energy monotonically decreases. However, the energy drop is not significant.

The effects of the topographic horizontal scale on the normalized power $\left\langle\widetilde{\mathcal{P}}_{n o}\right\rangle$ for all admissible harmonics and that is not affected by rotation (i.e. $n \in[1,7], \theta=0^{\circ}$ North) is also demonstrated on Figure 8 which illustrates the qualitative behavior of $\left\langle\widetilde{\mathcal{P}}_{n o}\right\rangle$ versus slope $\alpha$. For better visualization purposes, we use the "fill" option, set $\epsilon=0$ and use a larger stepsize $\Delta \alpha=10^{-4}$. It is crucial to observe in this figure that higher harmonic waves gradually die off from the wave field for larger values of $L$. We remark also, that although the maximum values of the normalized power $\left\langle\widetilde{\mathcal{P}}_{n o}\right\rangle$ look the same for smaller and larger values of $L$, in actuality it is not quite true; similarly to the observations made in Figure 7 for $n=1$, there is an insignificant drop of energy for all admissible modes $n$.

Figure 9 is used to show the results of numerical simulations for $\left\langle\widetilde{\mathcal{P}}_{n 10}\right\rangle$ versus $\alpha$, i.e, the normalized power for all admissible modes $n$ at latitude $\theta=10^{\circ}$ North for $L=1$. For better visualization purposes, we use again the "fill" option, set $\epsilon=10^{-5}$ and use the stepsize $\Delta \alpha=10^{-6}$. The more detailed behavior of the first-mode energy in the vicinity of the critical slope $\alpha_{n 10}^{c}$ is shown here. We observe in Figure 9 


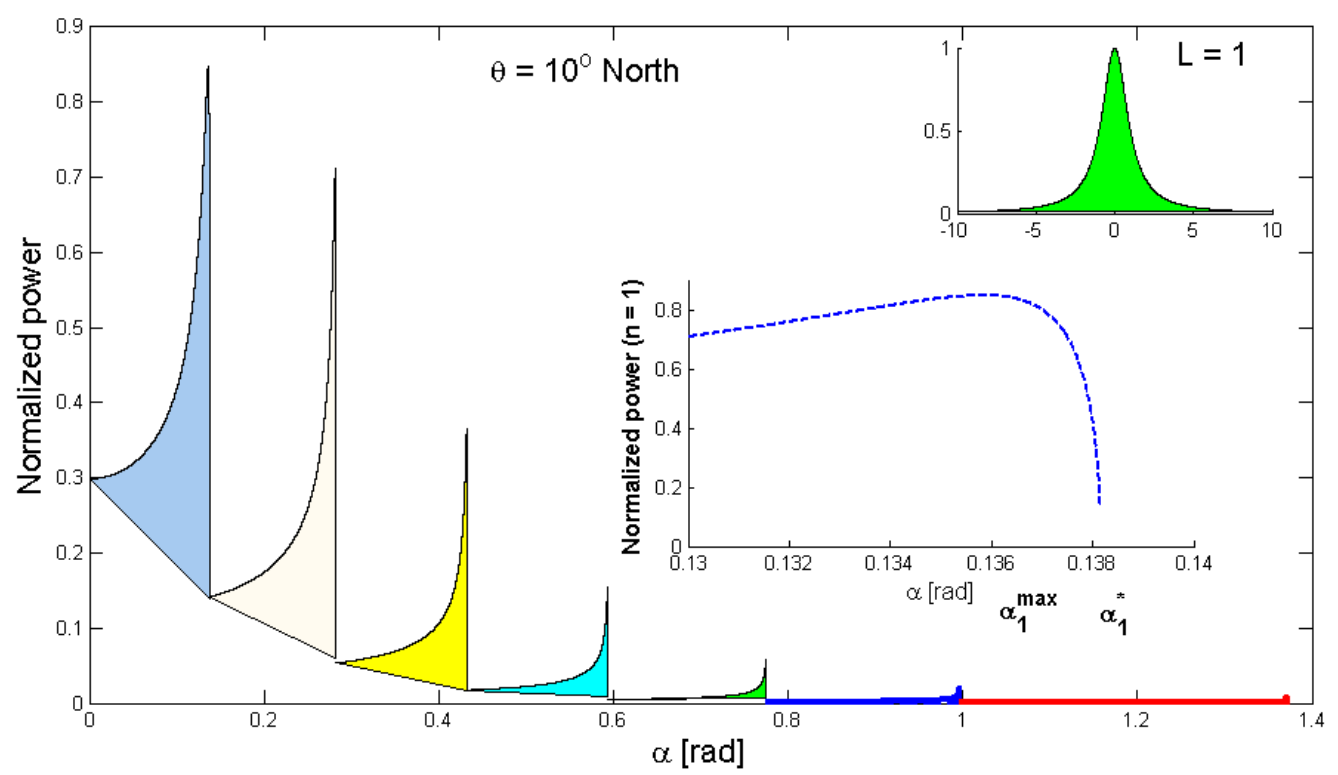

Figure 9. Energy flux at latitude $\theta=10^{\circ}$ North with $L=H$. Here we set $\epsilon=10^{-5}$ and stepsize $\Delta \alpha=10^{-6}$.
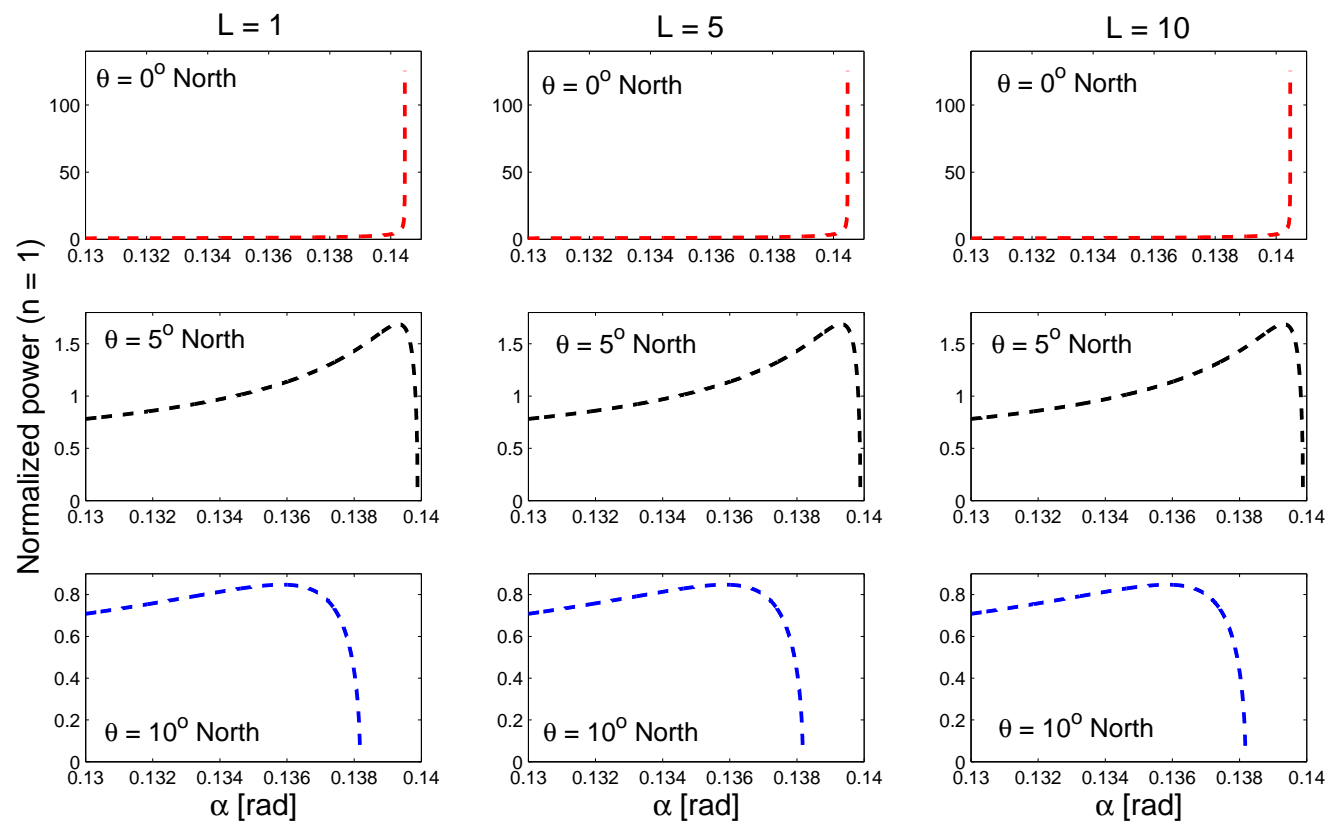

Figure 10. Analysis of the points of energy maximum $\alpha_{1}^{\max }$ and the approximation of the critical slope (i.e. the first zero $\alpha_{1}^{*}$ ) for the first-mode of the normalized power at different values of $L$ and latitude. 

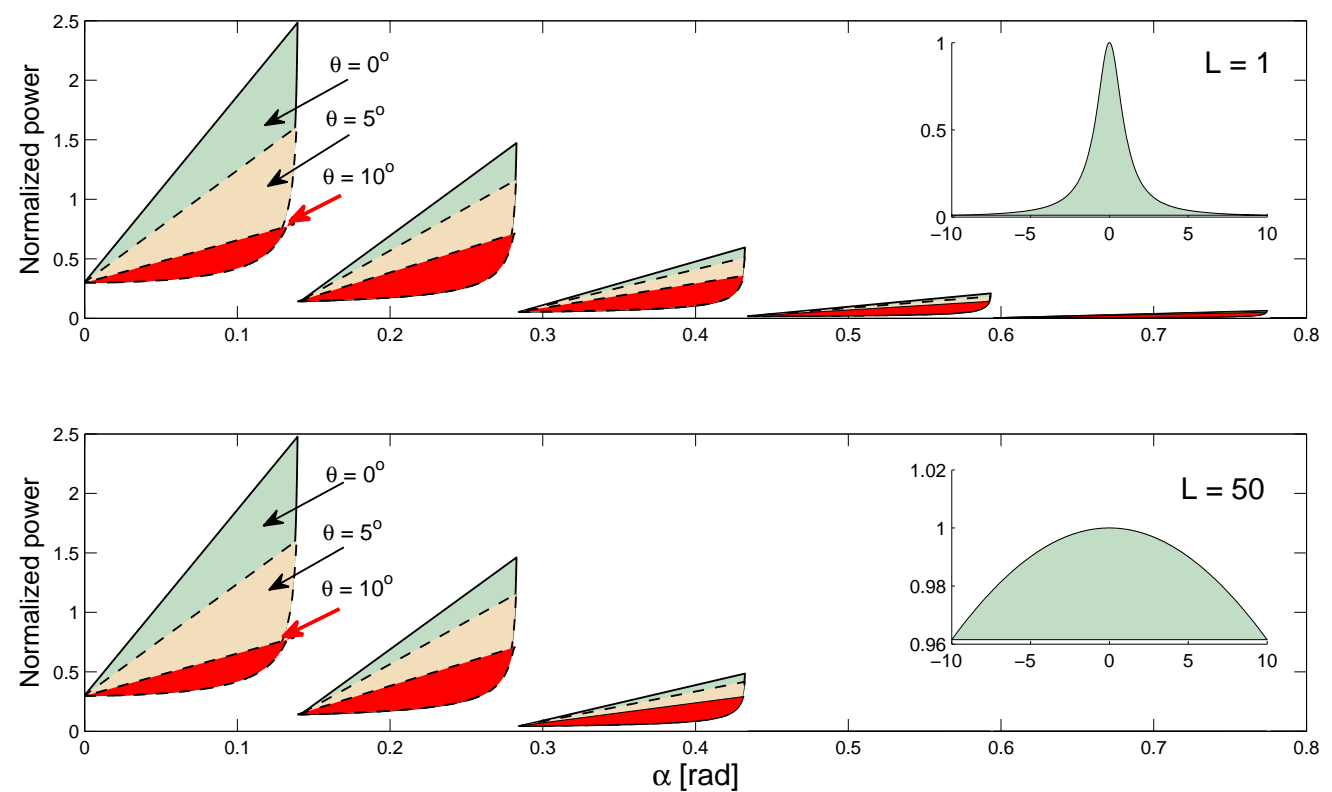

Figure 11. Effect of rotation on the energy flux at different values of the horizontal corrugation scale $L$.

that the energy attains its maximum not at this value of the slope but at some value $\alpha_{1}^{\max }<\alpha_{1}^{*} \approx \alpha_{1,10}^{c}$. Because of singularity, we cannot evaluate the energy at the critical slope $\alpha_{1,10}^{c}$ itself, so we associate the critical slope, say $\alpha_{1}^{*}$, which is reasonably close to $\alpha_{1,10}^{c}$.

Next, we investigate in more details the effects of rotation and the concavity of the topography on the resulting energy flux at the vicinity of the critical slopes $\alpha_{n, \theta}^{c}$. Figure 10 is used to present the results of numerical simulations describing the behavior of the first-mode normalized power $\left\langle\widetilde{\mathcal{P}}_{1 \theta}\right\rangle$ versus $\alpha$ for different values of latitude $\theta$ at the vicinity of $\alpha_{1, \theta}^{c}$. We can see from this list of plots, that for the given value of $\theta$, changing of the horizontal scale $L$ does not affect appreciably the power $\left\langle\widetilde{\mathcal{P}}_{1 \theta}\right\rangle$. However, for the fixed value of $L$, changes in latitude, have a noticeable impact on the power; not only on the numerical value at the vicinity of the critical slope $\alpha_{n, \theta}^{c}$, but also on the concavity of the curve, that describes the power. Similarly to the results shown in the detailed plot of Figure 9, we observe here that the energy attains its maximum not at the critical value of the slope but at some value $\alpha_{1}^{\max }<\alpha_{1}^{*} \approx \alpha_{1, \theta}^{c}$. For example, when $L=5$, we find that: at $\theta=5^{0}$, the critical slope is approximated by $\alpha_{1}^{*}=0.1399$ whereas the energy attains its maximum at $\alpha_{1}^{\max }=0.1393$ so that the difference between these two points is not zero and can be approximated by $\Delta \alpha^{*, \max } \approx 6.10 \times 10^{-4}$. Similarly, at latitude $\theta=10^{0}$, we find: $\alpha_{1}^{*}=0.1382$ and $\alpha_{1}^{\max }=0.1358$ so that $\Delta \alpha^{*, \max } \approx 2.4 \times 10^{-3}$.

Figure 11 shows (here, again, we use the "fill" option) the normalized power $\left\langle\widetilde{\mathcal{P}}_{n \theta}\right\rangle$ versus $\alpha$ for different values of latitude $\theta$ and different values of the horizontal topographic scale $L$. Particularly, we use $L=H$ (upper panel) and $L=50 H$ (lower panel). As has been demonstrated earlier in Figure 8, we observe the gradual dying of higher harmonic waves for larger values of $L$. In addition to the "dying off" phenomena, we observe that rotation tends to decrease the overall amount of radiating energy. So, we conclude that increasing the both latitude $\theta$ and the horizontal scale $L$ lead to the decrease of the 


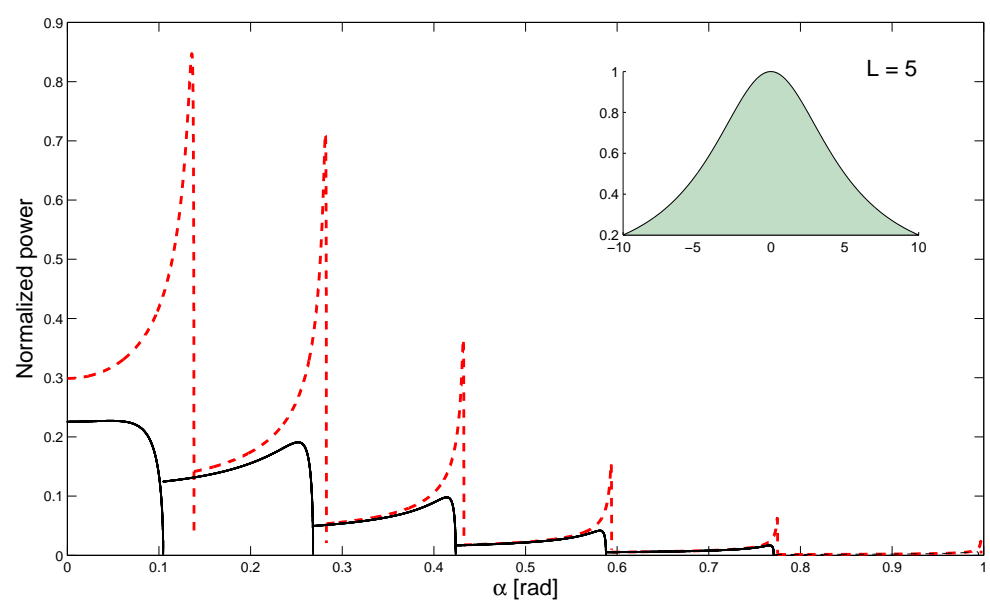

Figure 12. Comparison of the normalized energy flux at latitude $\theta=10^{\circ}$ North (red dashed line) with the flux at latitude $\theta=40^{0}$ North (close to critical latitude, plotted by a black pointed line) at the fixed value of $L=5$.

energy flux and removing the higher harmonics waves from the radiating internal wave field. Here we set $\epsilon=10^{-3}$ and stepsize $\Delta \alpha=10^{-4}$.

As an illustration to the latter conclusion about the effects of rotation, Figure 12 shows the overall behavior of the normalized power versus $\alpha$ at fixed horizontal scale $L=5 H$ and different values of latitude. In this particular example, shown in Figure 12, the normalized power at latitude $\theta=10^{\circ}$ North is plotted by red dashed line and the power at latitude $\theta=40^{\circ}$ North is plotted by by a black pointed line. Here we set $\epsilon=0$ and stepsize $\Delta \alpha=10^{-6}$.
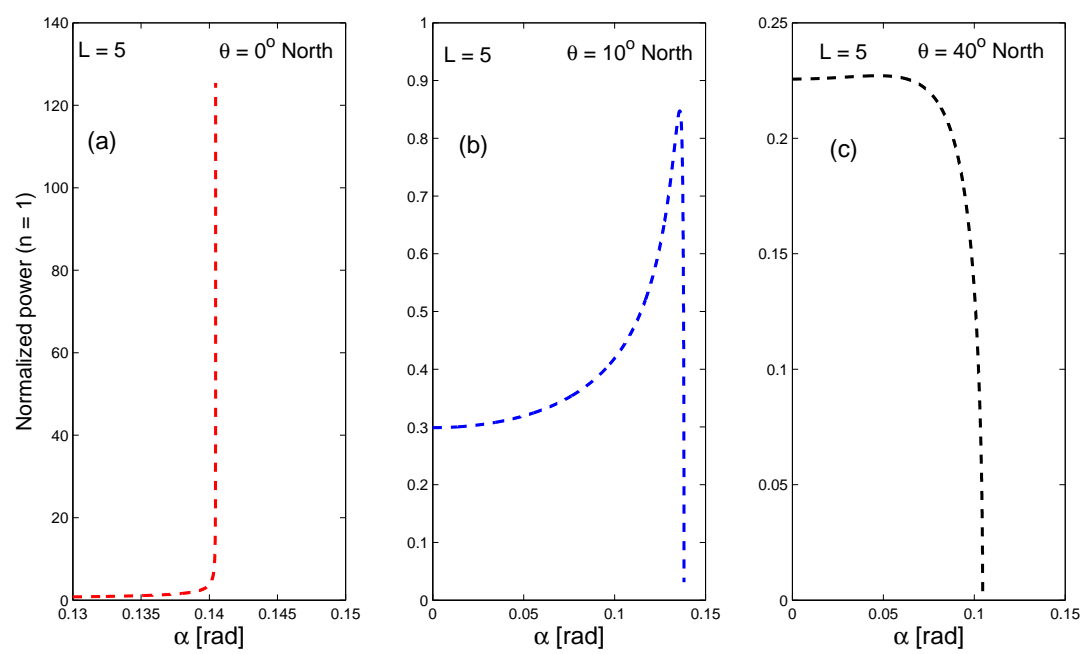

Figure 13. Analysis of the points of maximum $\alpha_{1}^{\max }$ and the first zero $\alpha_{1}^{*}$ for the firstmode of the normalized power at different values of latitude and fixed value of $L=5$. 
Finally, Figure 13 is used to visualize the effects of rotation on the resulting first-mode energy flux versus $\alpha$ for different values of latitude $\theta$ and the fixed horizontal topographic scale $L=5 H$ at the vicinity of the critical slopes $\alpha_{1, \theta}^{c}$. It is similar to the plot shown in Figure 10 but with the large range of latitudes. We can see from this list of plots, that the concavity of the curve, that describes the power, is more affected at smaller latitudes, somewhat in the range $\theta \in\left[0^{\circ}, 10^{\circ}\right]$ North but for larger values of latitudes, the concavity of the curve is not changed. Here we set $\epsilon=0$ and stepsize $\Delta \alpha=10^{-6}$. For example, in panel (b) we find: $\alpha_{1}^{*}=0.138$ and $\alpha_{1}^{\max }=0.1358$ so that $\Delta \alpha^{*, \max } \approx 2.2 \times 10^{-3}$. In panel (c) we find: $\alpha_{1}^{*}=0.1046$ and $\alpha_{1}^{\max }=0.047$ so that $\Delta \alpha^{*, \max } \approx 5.7 \times 10^{-2}$.

\section{Conclusions}

We have investigated the effects of the Earth's rotation and the topographic scales on the spectral problem associated with the radiating internal wave field that results from the reflection of an internal wave field imposed on the oscillatory background flow with a three-dimensional bottom topography. In this work, our focus was on a particular example in which the topography is represented by a continental slope with corrugation running up and down the slope, as shown schematically in Figure 14. In terms of mathematical tractability, it has been assumed in this work that dissipation processes are sufficiently strong to justify considering the ocean as infinitely deep fluid.

Special attention was given to estimating the flux of energy into the internal wave field in the vicinity of the critical slope

$$
\alpha \approx \alpha_{n \theta}^{c}=\arcsin \left(\frac{n^{2} \omega_{0}^{2}-f^{2}}{N^{2}-f^{2}}\right)^{\frac{1}{2}},
$$

in which

$$
s=\frac{k}{m}=\left(\frac{\omega^{2}-f^{2}}{N^{2}-\omega^{2}}\right)^{\frac{1}{2}}
$$

is the slope of an internal wave group velocity characteristic. This finding is in agreement with the suggestion of Legg, [25]. However, more detailed analysis shows that the radiating part of the energy attains its maximum not at the critical value of the slope but at some value $\alpha_{1}^{\max }<\alpha_{1}^{*} \approx \alpha_{1, \theta}^{c}$. This fact has not been remarked in Legg, [25] and has passed unnoticed in the previous studies in Ibragimov, [14].

It is found that the most efficient mixing in the deep ocean is due to reflection of an internal wave from a slope with the critical value given by (5.2). It is also found that an increasing in the both latitude and the topographic horizontal $L$ leads to the decreasing in the energy flux. Particularly, we observed the gradual dying off of higher harmonic waves for larger values of $L$. This means, that increasing the value $L$ leads to the decreasing of the value of the slope $\alpha_{n \theta}$ beyond which waves of tidal frequency are not generated. In addition to the "dying off" phenomena, we observe that rotation tends to decrease the overall amount of radiating energy.

We believe that, from physical point of view, the presence of critical slopes, after which, the higherharmonic waves are being dropped off the internal wave field can be explained by means of the previous relevant results in MacCready $\&$ Pawlak in Ref. [29], according to which, for the values of slope $\alpha$ exceeding the critical value, the fluid parcels do not have enough energy to go over the topography so that the fluid currents are trying to go around the topography.

The interest to this work has been motivated by the increasing interest of oceanographic community to the overpowering amount of untapped energy that is contained in the ocean, and especially in its coastal regions having a complex bathymetry, particularly the prominent corrugations, of the continental margin. Particularly, it is now recognized that the ocean can produce two main types of alternative energy: thermal energy from the sun's heat, and mechanical energy from the tides and waves ( see e.g. [42] and [7]). However, we are unaware of any theoretical, numerical or commercialized projects related to the available alternative energy due to internal waves in the coastal areas of the ocean. 

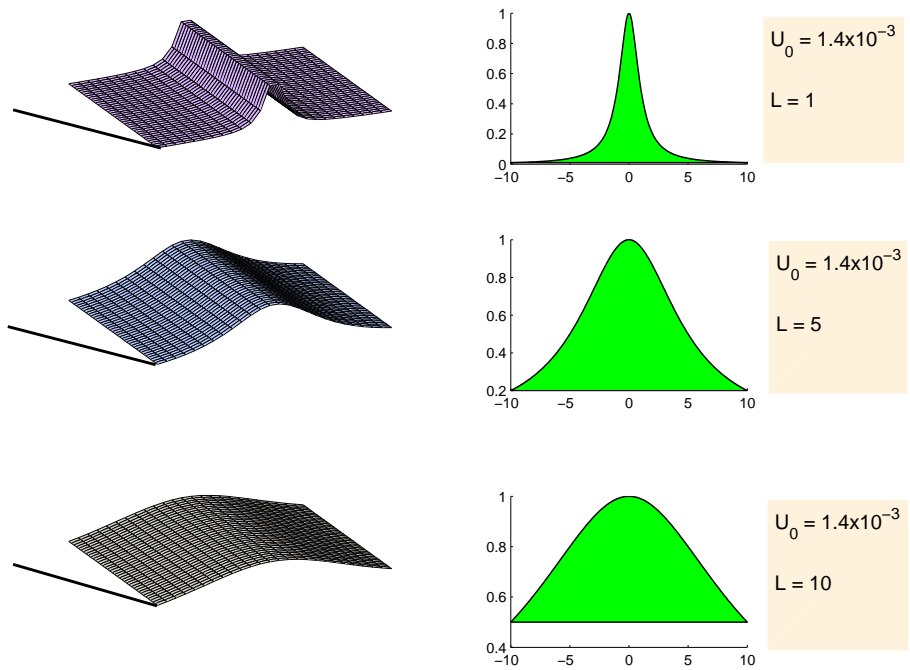

FIgURE 14. Schematic showing different horizontal scales $L$ of the corrugation geometry.

Acknowledgements. This research was supported in part by an appointment to the U.S. Department of Energy's Visiting Faculty Program.

This project has also been partially supported by the Office of Advance Scientific Computational Research of the US Department of Energy.

\section{References}

[1] C.H. Appenzeller, C.H. Davies, W.A. Norton. Fragmentation of stratospheric intrusions. J. Geophys. Res., 101 (1996), $1435-1456$.

[2] L. Armi. Effects of variation in eddy diffusivity on property distributions in the oceans. J. Mar. Res., 37 (1997), 515-530.

[3] P.G. Baines. Topographic Effects in Stratified Flows. Cambridge University Press. 1971.

[4] P.G. Baines. On internal tide generation models. Deep Sea Res., 29 (1982), 307-338.

[5] N.J. Balmforth, G.R. Ierley, W.R. Young. Tidal conversion by subcritical topography. J. Phys. Oceanogr., 32 (2002), 2900-2914.

[6] T.H. Bell. Lee waves in stratified flows with simple harmonic time dependence. J. Fluid Mech., 67 (1975), 705-722.

[7] R. Bedard, M. Previsic, G. Hagerman. North American Ocean Energy Status March 2007.Electric Power Research Institute (EPRI) Tidal Power (TP), Volume 8, G. 2007.

[8] G.D. Egbert, R. Ray. Signi cant dissipation of tidal energy in the deep ocean inferred from satellite altimeter data. Nature, 2000.

[9] N. Fraser. Surfing an oil rig. Energy Rev., (1999), 20-4 February/March.

[10] C. Garrett. Mixing with latitude. Nature, 422 (2003), 477-478.

[11] C. Garrett, P. MacCready, P.B. Rhines. Boundary mixing and arrested Ekman layers: Rotating, stratified flow near a sloping boundary. Annu. Rev. Fluid Mech., 25 (1993), 291-323.

[12] R. Grimshaw, N. Smyth. Resonant flow of a stratified fluid over topography. J. Fluid Mech., 169 (1986), $429-464$.

[13] A. Gill. Atmosphere-Ocean Dynamics. New York, etc., Academic Press., A., 1983.

[14] R.N. Ibragimov. Generation of internal tides by an oscillating background flow along a corrugated slope. Physica Scripta, 78 (2008), 065801.

[15] R. Ibragimov, N. Yilmaz, A. Bakhtiyarov. Experimental mixing parameterization due to multiphase fluid-structure interaction. Mech. Res. Comm., 38 (2011), 261-266.

[16] R.N. Ibragimov. Nonlinear viscous fluid patterns in a thin rotating spherical domain and applications. Phys. Fluids, 23 (2011), 123102.

[17] R.N. Ibragimov, V. Vatchev. Approximation of the Garrett-Munk internal wave spectrum. Phys. Let. A, 376 (2011), 94-101.

[18] R.N. Ibragimov, G. Jefferson, J. Carminati. Invariant and approximately invariant solutions of non-linear internal gravity waves forming a column of stratified fluid affected the Earth's rotation. Int. J. Non-Linear Mech., 51 (2013), 28-44. 
[19] J.C. Kaimal, J.J. Finnigan. Atmospheric Boundary Layer Flows. Their Structure and Measurement. Oxford University Press, 1994 London.

[20] L.H. Kantha, C.A. Clayson. Small Scale Processes in Geophysical Fluid Flows. New York, etc., Academic Press, International Geophysics Series, V. 67, 2000.

[21] S. Khatiwala. Generation of internal tides in an ocean of finite depth: analytical and numerical calculations. Deep See Res., I, 50 2003, 3-21.

[22] P.K. Kundu. Fluid Mechanics. Academic Press, Inc. 1990.

[23] E. Kunze, C. Garrett. Internal tide generation in the deep ocean. nnu. Rev. Fluid Mech, 39 (2007), 57-87.

[24] F. Lam, L. Mass, T. Gerkema. Spatial structure of tidal and residual currents as observed over the shelf break in the Bay of Biscay. Deep-See Res., I 51 (2004) 10751096.

[25] S. Legg, A. Adcroft. Internal wave breaking at concave and convex continental slopes. J. Phys. Oceanogr., 33 (2003), 2224-2247.

[26] S. Legg. Internal tides generated on a corrugated continental slope. Part 2: Along-slope barotropic forcing. J. Phys. Oceanogr., 34 (2004), no. 8, 1824-1838.

[27] S.G. Llewellyn Smith, W.R. Young. Conversion of the barotropic tide. J. Phys.Oceanogr., 32 (2002), $1554-1566$.

[28] R.R. Long. Finite amplitude disturbances in the flow of inviscid rotating and stratified fluids over an obstacle. Annu. Rev. Fluid Mech., 4 (1972), 69-92.

[29] P. MacCready, G. Pawlak. Stratified flow along a corrugated slope: Separation Drag and wave drag. J. Phys. Oceanogr., 31 (2001), 2824-2838.

[30] J.W. Miles. Waves and wave drag in stratified flows. Applied Mechanics: Proc. 12th Int.Cong. Appl. Mech., Springer, 1969.

[31] P. Muller, A. Naratov. The internal wave action model (IWAM). Proceedings, Aha Huliko'a Hawaiian Winter Workshop, School of Ocean and Earth Science and Technology, Special Publication, 2003.

[32] W. Munk, C. Wunsch Abyssal recipes II: energetics of tidal and wind mixing. Deep Sea Res., 45 (1998), $1977-2010$.

[33] J.C. Nappo. An introduction to atmospheric gravity waves. Academic Press, San Diego, 2002.

[34] J.D. Nash, J.M. Moum.Internal hydraulic flows on the continental shelf: High drag states over a small bank. Geophys. Res., 106 (2001), 4593-4612.

[35] G.T. Needler. Dispersion in the ocean by physical, geochemical and biological processes. Phil. Trans. R. Soc. London, A 319 (1986), 177-187.

[36] K.L. Polzin, J.M. Toole, J.R. Ledwell. Spatial variability of turbulent mixing in the abyssal ocean. Science, 276 (1997), 93-96.

[37] P. Queney. The problem of air flow over mountains: A summary of theoretical studies. Bull. Am. Meteorol. Soc. 29, 1948.

[38] R.S. Scorer. Environmental Aerodynamics, Halsted Press, N.-Y., 1978.

[39] S.A. Thorpe. The cross-slope transport of momentum by internal waves generated by alongslope currents over topography. J. Phys. Oceanogr., 26 (1996), 191-204.

[40] S.A. Thorpe. The generation of internal waves by flow over rough topography of continental slope. Proc. Roy. Soc. London A, 493 (1992), 115-130.

[41] J.H. Trowbridge, S.J. Lentz. Asymmetric behavior of an oceanic boundary layer above a sloping bottom. J. Pjys. Oceanogr., 21 (1991), 1171-1185.

[42] U.S. Department of Energy, 2009: Wind \& Hydropower Technologies Program. http://www1.eere.energy.gov/windandhydro/hydrokinetic/. Accessed April 2009.

[43] G.N. Watson. A Treatise on the Theory of Bessel Functions. 2nd ed., Cambridge University Press, 1966.

[44] C. Wunsch, R. Ferrari. Vertical mixing, energy, and the general circulation of the oceans. Annu. Rev. Fluid Mech., 36 (2004), 281-314.

[45] M.G. Wurtele, R.D. Sharman, A. Datta. Atmospheric lee waves". Annu. Rev. Fluid Mech., 28 (1996), $429-476$. 